\title{
L'influence sur les réseaux, une proposition de modélisation
}

\section{Influence over Networks, a modelling proposal}

\author{
Damien Nouvel ${ }^{1}$, Kévin Deturck ${ }^{1,2}$, Frédérique Segond $^{1,3}$, Namrata Patel $^{2}$
}

\author{
1 INALCO - 65, rue des Grands Moulins - Paris - France \\ prénom.nomeinalco.fr \\ 2 VISEO - 4, avenue Doyen Louis Weil - Grenoble - France \\ prénom.nomeviseo.com \\ ${ }^{3}$ Bertin IT - 10 bis, avenue Ampère - Montigny-le-Bretonneux - France \\ prénom.nom@bertin. fr
}

RÉSUMÉ. Cet article porte sur les influenceurs, définis comme des individus qui parviennent à impacter la prise de décision d'autres individus en interagissant avec eux. Avec le succès des médias sociaux cette dernière décennie, la détection de tels profils engendre un intérêt croissant dans des domaines aussi variés que le marketing, la défense ou encore le journalisme. Nous présentons dans cet article une nouvelle approche à la détection des influenceurs par une modélisation empirique du processus d'influence. Nous définissons d'abord le cadre théorique dans lequel nous avons conçu notre modèle. Nous décrivons ensuite la méthode employée pour déterminer les composantes générales de notre modèle et les caractériser par des traits linguistiques à partir des messages d'un média social. Nous abordons enfin l'objectif d'implémenter le modèle avec la création de données de référence.

ABSTRACT. This paper focuses on influencers, defined as individuals succeeding to have an impact on the decision process of other individuals simply through interaction. The success of social networks in the last decade led to an increasing interest for detecting such profiles. In such a context, we present a new influencer model based on the observation of real influence processes. We first define the theoretical frame in which we model the influence process. Then, we describe our empirical approach, based on the observation of influencers in forum discussions, allowing us to characterise each of our model component with linguistic features. Finally, we conclude by presenting, as a perspective, the model implementation with the linguistic feature annotation organised to acquire gold data.

MOTS-CLÉS : influenceurs, modélisation, approche empirique, traits linguistiques.

KEYWORDS: influencers, model, linguistic features, social media, social networks.

\section{Introduction}

Le développement des réseaux sociaux a fait émerger de nombreuses nouvelles problématiques liées à leur usage et leur impact dans la société. Il est indiscutable que les comportements des individus sont aujourd'hui largement liés à leurs interactions en ligne, aux connexions et communautés qu'ils établissent ainsi qu'au contenu qu'ils échangent.

Parmi ces problématiques, celle de l'influence est devenue ces dernières années le sujet de nombreuses recherches (Riquelme \& González-Cantergiani, 2016; Li et al., 2018). En effet, les éléments sur lesquels s'appuient une personne pour prendre des décisions en matière de consommation, de convictions politiques ou religieuses, sont largement liés aux contenus auxquels cette personne est exposée. Les enjeux sont importants et mieux comprendre comment s'établit l'influence, qui est influencé et qui est influenceur, sont autant de sujets que la numérisation de la société permet aujourd'hui de mieux étudier.

Dans ce qui suit, nous dressons un état de l'art sur l'influence (section 2), nous présentons la méthode de travail que nous avons suivie pour concevoir un nouveau modèle d'influenceur, en mettant l'accent sur son caractère empirique (section 3), nous décrivons notre modèle et les traits linguistiques identifiés pour caractériser sa première composante (section 4) puis nous discutons le modèle (section 5) et concluons (section 6). 


\section{2 État de l'art}

L'influence a d'abord été étudiée en sociologie et psychologie. En sociologie, (Katz \& Paul, 1955) étudient l'impact des relais de communication entre les médias et la population sur la formation des opinions. (Travers \& Milgram, 1977) introduisent une analyse structurelle des interactions dans un groupe social et mettent en évidence l'existence de certains individus charnières. (Freeman, 1978) formalise les premières mesures de centralité afin de quantifier l'importance structurelle des individus dans un réseau. La psychologie analyse comment les individus adoptent les comportements ou les opinions d'autrui lors de leurs interactions dans un groupe (Friedkin \& Johnsen, 1990).

Pour les réseaux sociaux virtuels, des approches adaptent la notion de centralité (Cha et al., 2010) et d'autres en analysent la dynamique (Borge-Holthoefer et al., 2012). D'autres approches, moins nombreuses, utilisent le traitement automatique des langues pour analyser le contenu linguistique des messages échangés entre les utilisateurs et y déceler les marqueurs linguistiques d'un discours influent (Biran et al., 2012; Rosenthal \& Mckeown, 2017). Ces approches se limitent à des sélections de traits théoriques et hypothétiques qui détectent des influenceurs potentiels plutôt que des influenceurs effectifs. C'est pourquoi notre approche propose une modélisation plus complexe portant sur l'ensemble du processus d'influence, avec une part importante dédiée aux effets de l'influence, pour que les individus détectés à l'origine soient effectivement des influenceurs.

\section{Méthodologie}

\subsection{Spécification théorique : définition d'un influenceur}

Avant le travail de modélisation, nous avons choisi une définition d'un influenceur qui corresponde aux intérêts soulevés par la détection automatique de ce profil dans les médias sociaux. Une application majeure des influenceurs dans ce type d'environnement est d'employer leur capacité à impacter les processus de prise de décision, que ce soit dans le cadre de campagnes politiques (Katz, 1957), commerciales (Trusov et al., 2010) ou de recrutement terroriste (Fernandez et al., 2018). Pour déterminer ce qu'est un influenceur, nous partons alors de la définition de l'influence telle que proposée par (Katz \& Paul, 1955) qui la décrit comme un processus social par lequel certains individus provoquent une prise de décision chez d'autres individus en interagissant avec eux. Nous définissons les influenceurs comme les individus qui sont à la source de ce processus.

Partant de l'idée que l'influence s'exerce au travers d'une communication médiée (dans notre cas écrite par l'entremise de réseaux sociaux), nous nous situons dans une vision où un influenceur est source d'éléments qui viennent modifier le comportement ou la pensée d'un autre individu. Nous reprenons à notre compte une vision inspirée de la psychologie sociale (Mason et al., 2007), qui caractérise les interactions entre individus comme des stimuli qui peuvent provoquer des changements dans l'état mental de l'individu les recevant et produire chez eux des décisions nouvelles.

\subsection{Spécification empirique : observation de phénomènes d'influence}

Pour nous assurer du bien-fondé de notre modèle d'influence, nous avons fait le choix de nous baser exclusivement sur l'observation d'un tel processus. Nous devions alors trouver un environnement d'observation qui mette en exergue des phénomènes d'influence. Puisque nous traitons d'un phénomène qui se produit entre des individus, les médias sociaux sont des environnements propices à notre étude.

Nous avons conduit nos observations sur le forum Change My View ${ }^{1}$ parce qu'il a des caractéristiques utiles à la conception empirique de notre modèle. Dans ce forum, l'auteur d'un fil de discussion expose son point de vue sur une thématique puis il demande aux lecteurs de le faire changer d'avis. Les intervenants qui parviennent à le faire changer d'avis sont explicitement désignés par ce dernier avec les raisons du changement. Ces intervenants particuliers correspondent d'autant plus à notre définition d'un influenceur qu'ils provoquent une décision nouvelle (un nouvel avis) chez des individus qui avaient déjà pris position. Les influenceurs sont bien mis en exergue par

1. https ://www.reddit.com/r/changemyview/ 
cette désignation de l'auteur initial qui constitue une annotation "ad hoc" en influence. Aussi, ce forum donne à voir la discussion ayant conduit à cette influence et donc les éléments du processus d'influence. De ce forum, un jeu de données a été extrait par (Tan et al., 2016). Il contient plus de 20000 fils de discussion pour plus d'un million de messages et plus de 80000 participants uniques. Nous avons alors opéré de façon empirique en sélectionnant au hasard un sous-ensemble de dix fils de discussion parmi ceux contenant au moins un changement d'avis. Notre environnement d'observation ainsi constitué, nous avons observé chaque phénomène d'influence identifié par une annotation "ad-hoc" afin de caractériser le processus afférent. Puisque nos observations ont porté sur des données langagières écrites, la spécification de notre modèle n’inclut que des traits linguistiques.

\subsection{Des premières sessions d'annotation pour l'implémentation du modèle}

Dans l'optique de développer un système de détection automatique d'influenceurs fondé sur notre modèle, nous avons besoin de données de référence pour (1) développer des règles linguistiques, entrainer des modèles par apprentissage et (2) évaluer les différents modules du système. Notre système va se composer de modules de détection (par règle et par apprentissage) pour chacun des traits linguistiques spécifiant les composantes du modèle.

Nous avons travaillé à l'annotation des expressions qui correspondent aux traits linguistiques dans des messages du forum Change My View ainsi que des tweets d'individus radicalisés dans le cadre du projet Trivalent ${ }^{2}$, pour lequel nous avons proposé de mettre en évidence les individus qui, en plus d'être radicalisés, sont des influenceurs. Nous avons commencé la rédaction d'un guide d'annotation qui définit pour le moment les traits de la première composante de notre modèle (cf. section 4.2.).

\section{Description du modèle}

\subsection{Conceptualisation générale}

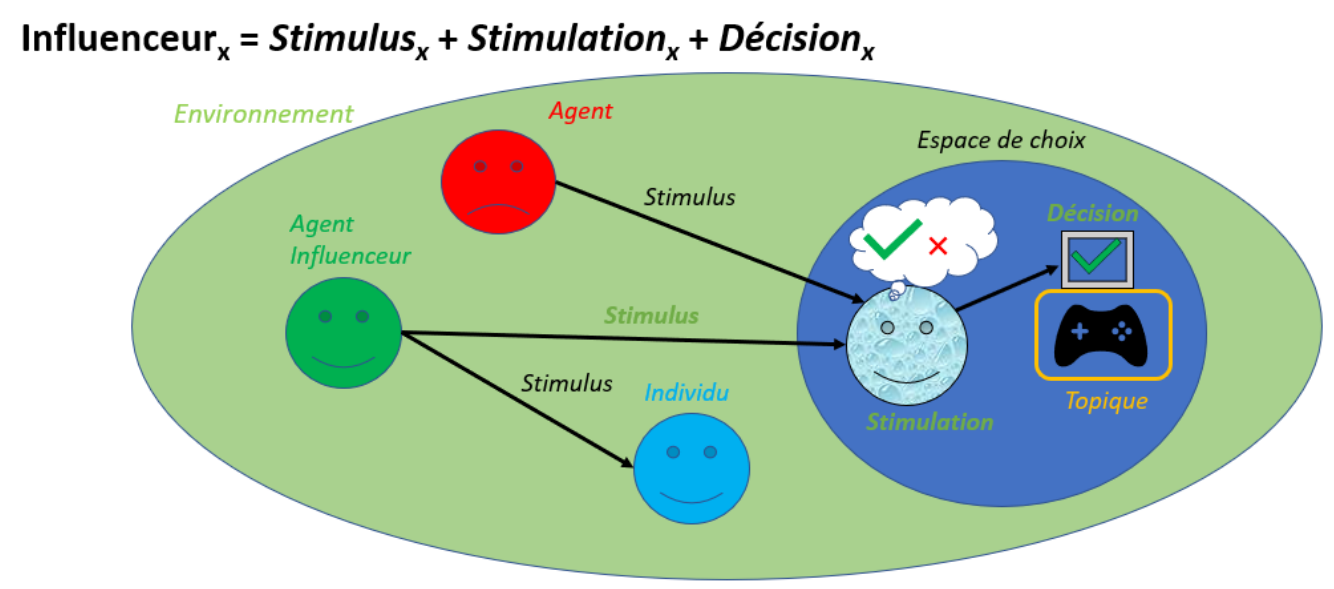

FIGURE 4.1. Modélisation de l'influence

La structure générale de notre modèle représente un influenceur comme la source d'un processus en trois étapes (Figure 4.1).

\section{Première étape : l'envoi d'un stimulus}

C'est le principe actif d'un influenceur. Un agent influenceur produit un stimulus qui agit sur la psychologie d'un individu et le conduit à prendre une décision nouvelle. Nous mentionnons et définissons les traits linguistiques réalisant cette composante en section 4.2.

\section{Deuxième étape : la stimulation}

Il s'agit d'une modification de l'état affectif ou cognitif d'un individu, déclenché par la réception d'un stimulus. Nous avons observé ce phénomène sous quatre catégories. 
- l'acquisition de connaissances,

- la génération de sentiments,

- la génération d'une compréhension,

- l'accord.

\section{Troisième étape : la prise d'une décision}

Un individu est un influenceur s'il parvient finalement à avoir un impact sur la prise de décision d'un autre individu. Dans nos données, la prise de décision se réalise lorsqu'un auteur exprime son changement d'avis. Un tel phénomène induit une nouvelle décision en tant que nouveau jugement sur un topique.

\subsection{Les types de stimuli identifiés dans les données}

Nous avons manuellement analysé le discours de messages ayant agi commme des stimuli afin d'en concevoir une typologie empirique. Sur la base de nos observations, nous avons identifié trois types de discours.

\subsubsection{Claim}

Un claim est une expression linguistique qui doit obligatoirement comporter les traits de factualité suivants :

— une interprétation objective (ce n'est pas une opinion),

- une forme affirmative,

— une forme descriptive (par exemple avec des entités nommées, des compléments circonstanciels).

Exemple

« Salman hold talks with Putin, a leader of crusaders »

Dans ce message, nous pouvons relever deux informations :

— «Salman hold talks with Putin » : ici, l'information porte sur un évènement

— «Putin, a leader of crusaders » : une information n'est pas nécessairement une proposition

La première information ne pose pas de problème puisqu'elle n'est portée que par des termes dont le sens ne relève pas d'un point de vue. La seconde information est un peu plus délicate parce qu'elle comporte le terme « crusaders » qui relève possiblement du point de vue de l'auteur. Toutefois, cela n'empêche pas une interprétation factuelle de l'expression « a leader of crusaders », Vladimir Poutine faisant partie des dirigeants occidentaux.

\subsubsection{Pédagogie}

La pédagogie est le discours d'un individu qui guide autrui dans sa compréhension du monde ou son comportement dans le monde. Ce type de discours prend la forme d'une explication ou d'un conseil. L'explication comporte des faits prétendus sur le monde, possiblement illustrés par des exemples, et une rhétorique logique qui structure le discours. Le conseil contient des suggestions ou des recommandations avec des verbes au conditionnel ou à l'impératif. Un discours pédagogique inclut bien souvent un destinataire (celui qu'il veut guider) avec notamment l'emploi de la deuxième personne.

\section{Exemple}

« Turn it off so they can stay in the darkness of their misguidance. »

On relève dans ce message la présence d'une recommandation exprimée à l'impératif : «Turn it off ». Cette recommandation est accompagnée d'une explication qui lui est subordonnée : "so they can stay in the darkness of their misguidance ». L'ensemble forme bien un discours pédagogique. 
L'argumentation est l'expression d'un lien logique entre un jugement et ses raisons. Le jugement est une description subjective de la réalité. Les raisons peuvent être des faits ou des jugements et sont présupposés vrais par l'auteur. Par sa rhétorique, une argumentation peut mettre en avant sa logique afin de soutenir sa validité. Notez que nous ne jugeons pas de la validité d'une argumentation ni de la véracité des allégations utilisées pour annoter le contenu argumentatif.

\section{Exemple}

«It appears that ISIS are the best diplomats on Earth since they work for Iran, America, Turkey, Saudi and Israel »

Dans cet exemple, nous trouvons un jugement : «ISIS are the best diplomats on Earth », porté par le superlatif « the best ». Ce jugement se veut factuel : «It appears that ». Il est lié logiquement par le marqueur discursif since à la proposition qui contient sa raison et qui est l'expression d'un claim : « they work for Iran, America, Turkey, Saudi and Israel ».

\section{Discussion}

Nous avons fait le choix d'une approche empirique pour nous assurer du bien-fondé de notre modèle et nous ne le tenons pas pour exhaustif puisqu'il est circonscrit par le champ de nos observations. Nos observations des influenceurs portent jusqu'à présent sur un seul jeu de données et les traits qui en découlent sont probablement orientés par la teneur des interactions qui ont lieu sur le média social correspondant. Étendre nos observations à un jeu de données Twitter nous permet de retrouver les traits linguistiques identifiés dans le forum à travers un genre différent. Toutefois, ce jeu de données, parce qu'il contient des messages indépendants de toute conversation, ne nous permet pas d'observer de nouveaux processus d'influence pour Twitter. L'apport pour la généricité de notre modèle est en cela limité.

Les premières sessions d'annotation que nous avons mises en place pour les traits linguistiques présentés en 4.2. mettent en évidence les difficultés afférentes à leur détection. Une difficulté majeure pour l'identification des traits est qu'ils reposent sur la compréhension voire l'interprétation des textes, donc sur de l'analyse sémantique. Pour le claim, les annotateurs peinent souvent à différencier l'expression d'un fait pur d'une expression d'opinion. La description de faits est pourtant l'essence même d'un claim. L'argumentation est, quant à elle, souvent confondue avec une opinion seule, or, son pouvoir d'influence réside précisémment dans sa capacité à justifier une opinion. Concernant le trait de pédagogie, il est souvent confondu avec les deux précédents traits. La confusion avec le claim vient du fait que la pédagogie peut utiliser des claims mais elle doit en comporter l'explication ou s'en servir pour expliquer un autre fait. Nous expliquons la confusion avec l'argumentation en ce que les deux traits peuvent expliquer quelque chose. La différence est que la pédagogie explique un fait quand l'argumentation explique une opinion. Nous précisons le contenu du guide d'annotation d'après ces observations afin de faciliter le travail des annotateurs par là améliorer la qualité des annotations.

\section{Conclusion}

Nous avons proposé dans cet article un nouveau modèle pour identifier un processus d'influence et, par là, les influenceurs qui en sont à l'origine. Ce nouveau modèle est fondé sur l'observation de phénomènes d'influence dans un forum de débat. Cela nous a permis de caractériser le comportement linguistique des influenceurs et de spécifier notre modèle par différents types de discours. Nous avons abordé la perspective d'implémenter notre modèle avec l'annotation des traits linguistiques qui le spécifient. Nous travaillons maintenant à la spécification linguistique de l'ensemble des composantes du modèle dans le but de les détecter automatiquement. 


\section{Références}

Biran O., Rosenthal S., Andreas J., McKeown K. \& Rambow O. (2012). Detecting influencers in written online conversations. In Proceedings of the Second Workshop on Language in Social Media, p. 37-45 : Association for Computational Linguistics.

Borge-Holthoefer J., Rivero A. \& Moreno Y. (2012). Locating privileged spreaders on an online social network. Physical review E, 85(6), 066123.

Cha M., Haddadi H., Benevenuto F. \& Gummadi K. P. (2010). Measuring user influence in twitter: The million follower fallacy. In fourth international AAAI conference on weblogs and social media.

FERnANDEZ M., ASIF M. \& Alani H. (2018). Understanding the roots of radicalisation on twitter.

FreEMAn L. C. (1978). Centrality in social networks conceptual clarification. Social networks, 1(3), 215-239.

Fried Kin N. E. \& Johnsen E. C. (1990). Social influence and opinions. Journal of Mathematical Sociology, 15(3-4), 193-206.

Katz E. (1957). The two-step flow of communication : An up-to-date report on an hypothesis. Public opinion quarterly, 21(1), 61-78.

Katz E. \& PAul F. (1955). Lazarsfeld (1955), personal influence. The Part Played by People in the Flow of Mass Communication. New York.

Li Y., FAn J., WANG Y. \& TAN K.-L. (2018). Influence maximization on social graphs : A survey. IEEE Transactions on Knowledge and Data Engineering, 30(10), 1852-1872.

Mason W. A., Conrey F. R. \& Smith E. R. (2007). Situating social influence processes : Dynamic, multidirectional flows of influence within social networks. Personality and social psychology review, 11(3), 279-300.

RiQuelme F. \& GonZÁLEZ-CANTERgiani P. (2016). Measuring user influence on twitter : A survey. Information Processing \& Management, 52(5), 949-975.

Rosenthal S. \& MCKeOWn K. (2017). Detecting influencers in multiple online genres. ACM Transactions on Internet Technology (TOIT), 17(2), 12.

TAn C., Niculae V., Danescu-Niculescu-Mizil C. \& Lee L. (2016). Winning arguments : Interaction dynamics and persuasion strategies in good-faith online discussions. In Proceedings of the 25th international conference on world wide web, p. 613-624 : International World Wide Web Conferences Steering Committee.

Travers J. \& Milgram S. (1977). An experimental study of the small world problem. In Social Networks, p. 179-197. Elsevier.

Trusov M., Bodapati A. V. \& Bucklin R. E. (2010). Determining influential users in internet social networks. Journal of Marketing Research, 47(4), 643-658. 